\title{
Limiar de dor à pressão em crianças com cefaleia*
}

\author{
Pressure pain threshold in children with headache
}

Gabriela Natália Ferracini ${ }^{1}$, José Geraldo Speciali²

* Recebido da Faculdade de Medicina de Ribeirão Preto da Universidade de São Paulo (FMRP-USP). Ribeirão

Preto, SP.

\section{RESUMO}

JUSTIFICATIVA E OBJETIVOS: A cefaleia está entre as dores mais comuns na infância, e estas apresentam mais sensibilidade a dor nas superfícies corporais, que pode ser avaliado pela algometria. O objetivo deste estudo foi rever na literatura os estudos que avaliaram o limiar de dor a pressão (LDP) em crianças com e sem cefaleia.

CONTEÚDO: Conduziu-se uma busca nos bancos de dados bibliográficos, incluindo Pubmed (National Library of Medicine), LILACS (Literatura Latino-Americana e do Caribe em Ciências da Saúde) e ADOLEC (Saúde na Adolescência) para identificar estudos científicos relevantes. Quatro estudos preencheram todos os critérios de seleção, totalizando 492 crianças e adolescentes, de 5 a 15,8 anos de idade. Destas, 75 tinham migrânea, 61 cefaleia tipo tensional e 356 sem histórico de cefaleia.

CONCLUSÃO: Não há consenso sobre a influência das cefaleias no LDP em crianças. Devido à escassez de estudos, não se pode concluir que o limiar de dor à pressão é menor em crianças com cefaleia quando comparado com crianças saudáveis.

Descritores: Cefaleia, Crianças, Limiar de dor.

1. Fisioterapeuta pela Universidade Paulista de São José do Rio Preto; Mestranda em Neurociências da Faculdade de Medicina de Ribeirão Preto (FMRP-USP). Ribeirão Preto, SP, Brasil.

2. Médico Neurologista, Professor-Associado de Neurologia do Departamento de Neurologia, Psiquiatria e Psicologia Médica da Faculdade de Medicina de Ribeirão Preto (FMRP-USP). Ribeirão Preto, SP, Brasil.

Endereço para correspondência:

Prof. Dr. José Geraldo Speciali

Avenida dos Bandeirantes, 3.900

14049-900 Ribeirão Preto, SP.

E-mail: speciali@netsite.com.br

\section{SUMMARY}

BACKGROUND AND OBJECTIVES: Headache is one of the most common pains during childhood and children have higher sensitivity to pain in body surfaces, which can be evaluated by algometry. This study aimed at reviewing in the literature studies evaluating pressure pain threshold (PPT) in children with and without headache.

CONTENTS: Bibliographic databases were searched, including Pubmed (National Library of Medicine), LILACS (Latin-American and the Caribbean Literature on Health Sciences) and ADOLEC (Health during Adolescence) to identify relevant scientific studies. Four studies met all inclusion criteria with a total of 492 children and adolescents, aged from 5 to 15.8 years. From them, 75 had migraine, 61 had tension headache and 356 had no headache history.

CONCLUSION: There is no consensus about headache influence on PPT in children. Due to the scarcity of studies, one could not conclude that pressure pain threshold is lower in children with headache as compared to healthy children.

Keywords: Children, Headache, Pain threshold.

\section{INTRODUÇÃO}

A cefaleia está entre as dores mais comuns na infância. Dentre as cefaleias, a migrânea é a principal e acomete $5 \%$ a $10 \%$ de crianças e adolescentes, sua frequência aumenta com a idade, predomina nos meninos até os 7 anos, iguala-se na faixa etária de 7 a 11 anos e posteriormente acomete mais meninas. Nas crianças, assim como nos adultos, a migrânea sem aura é a mais frequente ${ }^{1,2}$.

Os critérios diagnósticos segundo a Classificação Internacional das Cefaleias (2004) (ICHD-II) para a cefaleia migranosa na infância só diferem do adulto em relação à duração mínima da fase dolorosa. As 
crises de cefaleia migranosa na infância são de curta duração (1-4 horas) e, mais frequentemente que nos adultos, localizadas bilateralmente ${ }^{3}$.

Crianças com cefaleia apresentam mais sensibilidade à dor nas superfícies corporais do que crianças saudáveis, uma forma de avaliar esta alteração é com a utilização da algometria ${ }^{4}$.

A algometria é uma técnica que quantifica a capacidade de percepção e de tolerância dolorosa através da pressão sobre os nociceptores (limiar de dor a pressão). O limiar de dor a pressão é a menor pressão capaz de gerar dor ou desconforto, sendo influenciado pela idade, sexo e regiões do corpo $^{5,6}$.

Crianças com limiares baixos sentem mais dor, e estímulos de intensidade que não causariam dor, tornam-se dolorosos. Essas alterações na faixa etária pediátrica devem ser observadas, e se constatadas, medidas que elevem o limiar de dor deverão ser incorporadas pelo profissional antes de qualquer procedimento que apresente risco de dor ${ }^{7,8}$.

Devido à importância e a pouca divulgação dos conhecimentos sobre limiar de dor em crianças, o objetivo deste estudo foi rever na literatura a cefaleia em crianças.

\section{DESENVOLVIMENTO}

Foi conduzida uma busca em banco de dados computadorizado para identificar artigos científicos relevantes para o estudo. Os artigos foram selecionados entre 1993 e 2010 e obtidos em buscas em banco de dados bibliográficos, incluindo Pubmed (National Library of Medicine), LILACS (Literatura Latino-Americana e do Caribe em Ciências da Saúde) e ADOLEC (Saúde na Adolescência).
Foram incluídos os estudos clínicos, de coorte e casos controlados com dados epidemiológicos das amostras. E excluídos os relatos de casos e revisões da literatura.

Os critérios de inclusão dos artigos foram: casuística com idade entre 4 e 16 anos, avaliação do limiar de dor à pressão em crianças sem histórico de cefaleia e em crianças com diagnóstico de cefaleia segundo os critérios de 1988 e 2004 da Sociedade Internacional de Cefaleia (IHC-II) e utilização da algometria como método de avaliação do limiar de dor a pressão. Os artigos foram selecionados a partir dos dados contidos no resumo ou, nos casos em que o resumo não estava disponível, por meio das informações contidas em seu título.

\section{RESULTADOS}

O levantamento de dados resultou no total de 47 artigos científicos. Destes, 13 foram selecionados como artigos em potencial para o estudo, baseado em seu resumo. Dentre os artigos selecionados para o estudo, nove foram descartados. Os motivos para a exclusão foram: não objetivaram avaliar o limiar de dor a pressão (LDP) pelo método de algometria, não havia o relato do critério diagnóstico para cefaleia. Quatro artigos preencheram todos os critérios de seleção, totalizando 492 crianças e adolescentes, de 5 a 15,8 anos de idade. Destas, 75 tinham migrânea, 61 cefaleia tipo tensional e 356 sem histórico de cefaleia (Tabela 1).

O LDP foi menor nas meninas e nas faixas etárias menores. As crianças com cefaleia apresentaram limiar de dor a pressão menor do que as saudáveis ${ }^{4,8}$ (Tabela 2). Todos os estudos avaliados apresentavam limitações científicas serem não encobertos e não controlados.

Tabela 1 - Estudos encontrados nos diferentes bancos de dados

\begin{tabular}{llccc}
\hline Banco de Dados & Descritores & Resultados & $\begin{array}{c}\text { Artigos } \\
\text { Selecionados }\end{array}$ & $\begin{array}{c}\text { Artigos } \\
\text { Incluídos }\end{array}$ \\
\hline \multirow{2}{*}{ Pubmed } & LDP em crianças & 41 & 10 & 2 \\
& LDP em crianças com cefaleia & 3 & 3 & 2 \\
\multirow{2}{*}{ Adolec } & LDP em crianças & 2 & - & - \\
& LDP em crianças com cefaleia & - & - & - \\
Lilacs & LDP em crianças & 1 & - & - \\
Total & LDP em crianças com cefaleia & - & - & 4 \\
\hline
\end{tabular}

LDP $=$ Limiar de dor a pressão 
Tabela 2 - Estudos que avaliaram o limiar de dor a pressão em crianças com e sem cefaleia

\begin{tabular}{|c|c|c|c|}
\hline Estudos & País/Local & Amostra & Resultados \\
\hline Metsahonkala e col. ${ }^{4}$ & Turku/Escola primária. & $\begin{array}{l}168 \text { crianças, } 48 \text { com } \\
\text { migrânea, } 61 \text { com CTTE e } 59 \\
\text { sem cefaleia. }\end{array}$ & $\begin{array}{l}\text { O LDP foi menor em } \\
\text { meninas do que em meninos } \\
\text { em todos os grupos. } \\
\text { Não houve diferença do LDP } \\
\text { entre os grupos. }\end{array}$ \\
\hline Hogeweg e col. ${ }^{5}$ & Holanda/ 3 escolas primárias. & $\begin{array}{l}69 \text { crianças, sem histórico de } \\
\text { cefaleia }\end{array}$ & $\begin{array}{l}\text { LDP na região paraespinhal } \\
\text { foi maior de rostral para } \\
\text { caudal; } \\
\text { LDP nas regiões periféricas } \\
\text { foi maior do que na } \\
\text { paraespinhal, exceto } \\
\text { tornozelos. }\end{array}$ \\
\hline Zohsel e col. ${ }^{8}$ & $\begin{array}{l}\text { Germânia/ Hospital da } \\
\text { Universidade de Heidelberg. }\end{array}$ & $\begin{array}{l}55 \text { crianças, } 27 \text { com migrânea } \\
\text { e } 28 \text { sem cefaleia. }\end{array}$ & $\begin{array}{l}\text { Crianças migranosas } \\
\text { apresentaram menor limiar de } \\
\text { dor à pressão. }\end{array}$ \\
\hline Duarte, Goulart e Penna ${ }^{9}$ & $\begin{array}{l}\text { Minas Gerais/ Hospital } \\
\text { das Clínicas da Faculdade } \\
\text { Federal. }\end{array}$ & $\begin{array}{l}200 \text { crianças, sem histórico } \\
\text { de cefaleia. }\end{array}$ & $\begin{array}{l}\text { LDP foi menor nas crianças } \\
\text { do que nos adolescentes, em } \\
\text { todas as regiões avaliadas. }\end{array}$ \\
\hline
\end{tabular}

$\mathrm{CTTE}=$ cefaleia tipo tensional episódica; $\mathrm{LDP}=$ limiar de dor à pressão

\section{DISCUSSÃO}

No presente estudo observou-se que a literatura é muito deficiente em abordar o tema limiar de dor a pressão em crianças. Quando a busca foi dirigida para publicações que comparassem LDP em crianças com e sem cefaleia foram localizados apenas 2 estudos $^{4,8}$. A análise dessas publicações não permitiu conclusões, pois um deles não encontrou diferenças significativas entre os grupos com e sem cefaleia enquanto que o outro concluiu que crianças com migrânea têm um menor LDP.

O estudo que avaliou o LDP de 48 crianças com migrânea, 61 crianças com cefaleia tipo tensional episódica e 59 crianças sem cefaleia, de exclusivamente 13 anos, em 7 pontos cefálicos e 3 pontos extracefálicos, juntamente com a aplicação de questionário para avaliar a presença de outras dores ${ }^{4}$. Concluíram que crianças com migrânea relatam outras dores, como dor estomacal e na coluna lombar mais frequentemente do que as crianças com CTTE e as sem cefaleia. Porém, não houve diferença do LDP entre os três grupos avaliados.

Foi avaliado o limiar de dor por pressão e temperatura nas áreas trigeminal e região tenar bilaterais, de 25 crianças migranosas (5 com aura, $20 \mathrm{sem}$ aura) e 28 crianças sem cefaleia, de 9-15 $\operatorname{anos}^{8}$. As crianças migra- nosas apresentaram limiares de dor a pressão menor do que as crianças sem cefaleia, mas estes limiares foram maiores quando a mãe estava presente no momento da avaliação. Os autores concluíram que as meninas com migrânea são mais propensas a sensibilização central, o qual pode aumentar o risco de continuar sofrendo crises migranosas na idade adulta ${ }^{8}$.

Outro estudo ${ }^{9}$ que avaliou 100 crianças saudáveis, 45 meninos e 55 meninas de 5,3-15,8 anos, a fim de correlacionar o LDP e a idade. Foram avaliadas 17 áreas do corpo com um algômetro mecânico, na razão de $0,5 \mathrm{~kg} / \mathrm{cm}^{2} / \mathrm{s}$. Evidenciaram-se correlações positivas entre idade e LDP nos músculos trapézio, deltoides e supraespinhais, 9 áreas da parede abdominal e parte medial das tíbias. A idade do paciente deve ser considerada nas investigações e avaliação clínicas sobre dor em crianças e adolescentes. Concluiu-se que quanto menor a idade menor são os limiares. Avaliando o LDP em 12 áreas anatômicas de 69 crianças saudáveis, 33 garotos e 36 garotas com média de 11,4 anos, os autores não encontraram diferença significativa do LDP entre os sexos, o LDP aumentou com a idade na região paraespinhal, mas não nas extremidades, podendo ser influenciado pela consistência do tecido ${ }^{5}$. 
Crianças portadoras de alguma doença orgânica ou funcional apresentam redução do limiar de dor à pressão (LDP) em várias regiões do corpo, além das regiões dolorosas da própria doença. Existe uma associação entre dor abdominal recorrente e dor de crescimento e LDP diminuído, porém não foi encontrada uma relação de causalidade para esta ocorrência, sugerindo existir uma sensibilização central provocada pela recorrência da dor. A determinação do limiar de dor a pressão é uma técnica indireta, que provê valores quantitativos, permitindo o controle da velocidade e direção da pressão, a fim de estabelecer o comportamento de determinado grupo de nociceptores e, se estes estão sendo influenciados pelo sistema nervoso central pelas vias aferentes e eferentes ${ }^{8,9}$. A redução global do LDP nas superfícies corporais sugere uma alteração da modulação da dor, com fatores periféricos e centrais do sistema nervoso envolvidos na percepção da dor ${ }^{4,5,7}$. O limiar de percepção e de tolerância dolorosa de um determinado nociceptor está relacionado com a capacidade de captação nociceptiva e dos controles excitatórios e inibitórios superiores, assim, quando há um desequilíbrio nestes centros, estímulos subliminares produzem percepção dolorosa (alodínia) ou tolerância reduzida (hiperalgesia) ${ }^{4,6,9}$.

Devido à escassez de estudos, não se pode concluir que o limiar de dor a pressão é menor em crianças com cefaleia quando comparado com crianças saudáveis. A determinação dos limiares de dor a pressão é uma ferramenta útil que pode caracterizar um determinado grupo de pacientes e auxiliar na compreensão do comportamento álgico.

Estudos futuros devem ser direcionados envolvendo o LDP em crianças com cefaleia e sem histórico de cefaleia. E, dentro do grupo de crianças com cefaleia qual a importância da presença da redução do LDP na abordagem clínico-terapêutica desse grupo. No entanto a idade, o local examinado e a presença de doenças dolorosas associadas à cefaleia devem ser considerados quando da estruturação da metodologia aplicada, pois influenciam o LDP nessas faixas etárias.

\section{CONCLUSÃO}

Devido à escassez de estudos, não se pode concluir que o limiar de dor a pressão é menor em crianças com cefaleia quando comparado com crianças saudáveis.

\section{REFERÊNCIAS}

1. Puccini RF, Bresolin AB. Dores recorrentes na infância e adolescência. J. Pediatr 2003;79(Suppl 1):65-76.

2. Hershey AD, Winner PK. Pediatric migraine: recognition and treatment. J Am Osteopath Assoc 2005;105(4 Suppl 2):2S-8S.

3. The International Classifications of Headache Disorders. Headache Classification Subcommittee of the International Headache Society. $2^{\text {nd }}$ ed. Cephalalgia 2004;24(Suppl 1):9-160.

4. Metsahonkala L, Anttila P, Laimi K, et al. Extracephalic tenderness and pressure pain threshold in children with headache. Eur J Pain 2006;10(7):581-5.

5. Hogeweg JA, Kuis W, Oostendorp RA, et al. The influence of site of stimulation, age, and gender on pain threshold in healthy children. Phys Ther 1996;76(12):1331-9.

6. Duarte MA, Goulart EM, Penna FJ. Pressure pain threshold in children with recurrent abdominal pain. J Pediatr Gastroenterol Nutr 2000;31(3):280-5.

7. Hashkes PJ, Friedland O, Jaber L, et al. Decreased pain threshold in children with growing pains. J Rheumatol 2004;31(3):610-3.

8. Zohsel K, Hohmeister J, Oelkers-Ax R, et al. Quantitative sensory testing in children with migraine: preliminary evidence for enhanced sensitivity to painful stimuli especially in girls. Pain 2006;123(1-2):10-8.

9. Duarte MA, Goulart EM, Penna FJ. Pain threshold and age in childhood and adolescence. J Pediatr 1999;75(4):244-8.

Apresentado em 22 de março de 2011.

Aceito para publicação em 23 de agosto de 2011. 